\title{
Chilblain Lupus with Nail Involvement: A Case Report and a Brief Overview
}

\author{
Martina Lambertini Colombina Vincenzi Emi Dika Michelangelo La Placa \\ Dermatology Division, Department of Experimental, Diagnostic and Specialty Medicine, University of Bologna, \\ Bologna, Italy
}

\section{Established Facts}

- Chilblain lupus erythematosus is a rare type of cutaneous lupus erythematosus typically presenting with purplish papules and plaques on the extremities during cold seasons.

\section{Novel Insights}

- Severe onycholysis and nail plate destruction due to vascular impairment is a possible presenting sign in chilblain lupus erythematosus.

\section{Keywords}

Hair disorder - Nail disorder - Connective tissue . Chilblain lupus erythematosus · Lupus pernio · Nail fold capillaroscopy

\begin{abstract}
Chilblain lupus erythematosus is a rare, chronic variant of cutaneous lupus erythematosus that occurs during cold or damp periods on the hands, fingers, or feet. It is often associated with other forms of cutaneous lupus and about $20 \%$ of patients develop systemic lupus erythematosus. Various medications have been put forward, including steroids, mycophenolate mofetil, calcium channel blockers, and hydroxychloroquine, but the symptoms do not remit completely.

(c) 2018 S. Karger AG, Basel
\end{abstract}

\section{KARGER}

(c) 2018 S. Karger AG, Basel

E-Mail karger@karger.com

www.karger.com/sad

\section{Introduction}

Chilblain lupus erythematosus (CHLE) is a rare, chronic variant of lupus erythematosus (LE), sometimes associated with other forms of cutaneous (CLE) or systemic LE (SLE) $[1,2]$. We herein report the case of a Caucasian woman affected by discoid lupus erythematosus (DLE) of the scalp who developed a sporadic form of CHLE, with an overview on the cutaneous findings and most standardized therapeutic options.

\section{Case Report}

An 82-year-old woman consulted us in wintertime concerning dermatosis on the extremities that had suddenly appeared 2 months before. She had been under follow-up in our clinic for chronic DLE of the scalp for about 10 years and had been treated 
Fig. 1. Clinical features of patients with chilblain lupus erythematosus. a-c Erythematous/purplish papules and plaques on the sole and big toes. The nail plate is partially destroyed with hyperkeratosis of the nail bed. d Nail fold capillaroscopy from the left big toe showing tortuous capillaries.
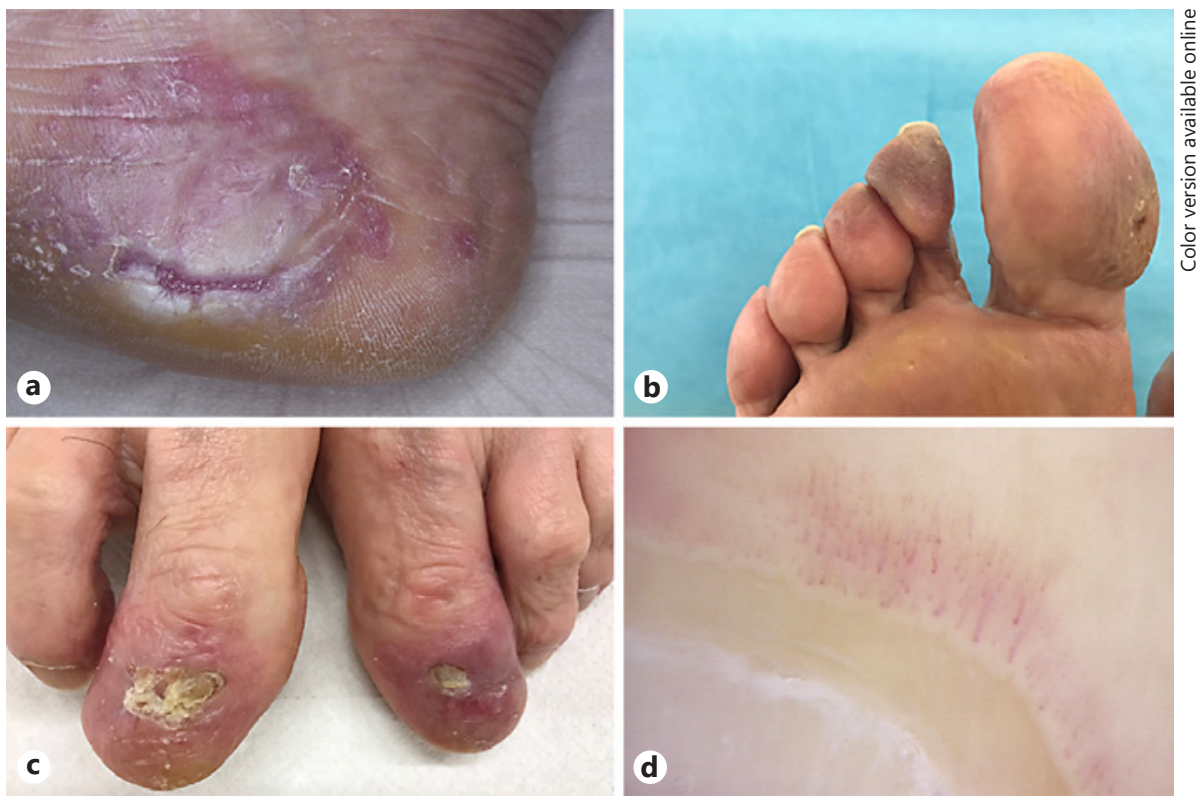

Fig. 2. Hematoxylin and eosin (H\&E) staining from lesional skin. a Thin epidermis with hyperkeratosis, orthokeratosis, and mixed infiltrate in the dermis. $\mathrm{H} \& \mathrm{E}$. $\times 20$. $\mathbf{b}$ Higher magnification view showing dermal interstitial and perivascular phlogosis with lymphocytic infiltrates with colloid bodies.
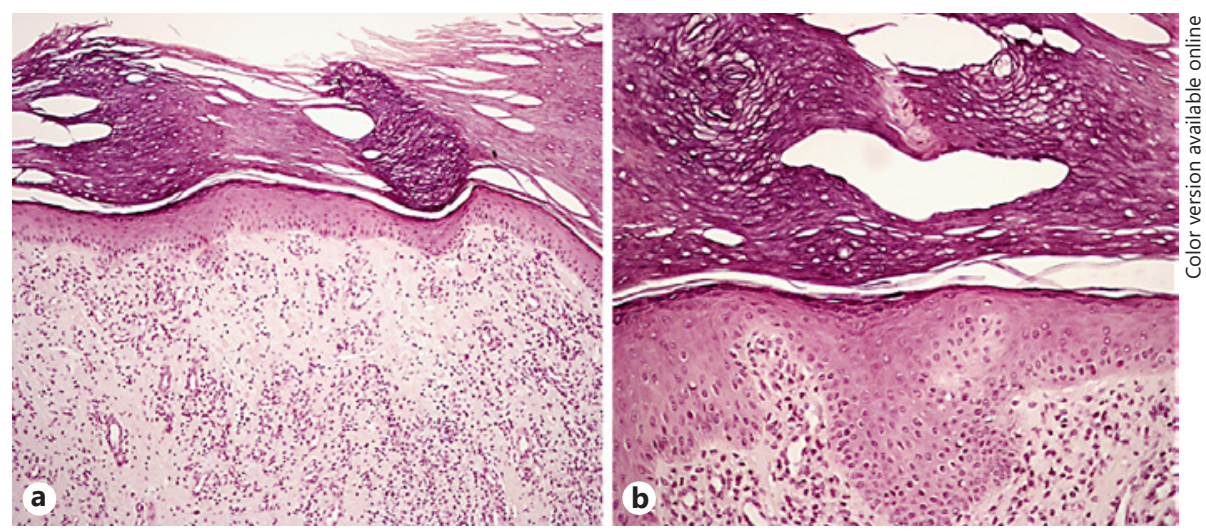

with hydroxychloroquine, topical steroids, and minoxidil $2 \%$ solution with partial benefit. Over the last year, hydroxychloroquine was discontinued due to retinal toxicity, so the patient continued the treatment with minoxidil solution with good results and stabilization of the disease. At the time of consultation, we observed bluish/purplish papules and plaques on the big toes and soles of both feet. The nail beds were atrophic and partially ulcerated, and the overlying nail plates were almost completely destroyed. Fine scaling was present on the heels and the patient reported mild pain. Nail fold capillaroscopy showed microvascular changes typically seen in connective tissue disorders, including tortuous capillaries, enlarged capillaries, and/or microhemorrhages (Fig. 1).

Laboratory tests revealed leukocytosis, elevation of the erythrocyte sedimentation rate $(>40)$, decreased level of albumin, and increase of $\alpha_{1^{-}}, \alpha_{2^{-}}$, and $\gamma$-globulins. The autoimmune profile showed positivity of Ro/SSA antibodies $(+++)$ and antinuclear antibodies (1:640, speckled pattern). A 4-mm punch biopsy from the plaque of the right heel was performed for histological analysis, leading to the diagnosis of LE (Fig. 2). Because of chronic DLE and considering the clinical and histological findings, we made the diagnosis of CHLE and started to treat the patient with topical tacrolimus, mycophenolate mofetil at the dosage of $500 \mathrm{mg}$ twice daily, and nifedipine $20 \mathrm{mg}$ daily. The patient did not fulfill the American Rheumatology Association (ARA) criteria for the diagnosis of SLE. At the 1-year follow-up, the cutaneous symptoms were still present, with mild improvement.

\section{Discussion}

CHLE is a rare variant of LE that presents as sporadic or as an autosomal inherited form, namely familial CHLE. The first symptoms generally occur during the cold seasons and worsen partially in damp conditions $[1,2]$. Clinically, CHLE presents as erythematous/purplish papules 
or plaques on the acral sites, both superior and inferior. Fingers and toes are the most involved sites, with ulceration and necrosis occurring in some patients; less frequently, palms and soles are involved with hyperkeratosis and/or fissuration. Other sites include the ears, the nose and, very rarely, the trunk. Itching may be an initial symptom, subsequently accompanied by pain. The ulcerations in toes and feet may develop scarring $[1,2]$. The incidence of CHLE has not been reported, although it is known that it may develop years or decades after the first clinical signs of CLE [2]. Millard and Rowell [3] described 17 cases of CHLE where 3 had concomitant SLE. Yell et al. [4] reported 15 cases $(20.5 \%)$ of CHLE in a large case series of SLE. Komori et al. [5] described an overlap syndrome with histological features of CHLE and lichen planus in the same lesion. Raynaud phenomenon has been seldom associated [6].

CHLE may be classified into sporadic and familial forms. The pathogenesis of sporadic CHLE is not fully known but seems to be related to impairment of the microcirculation, determining blood stasis and vascular occlusion exacerbated by cold temperatures [2]. The positivity of Ro/SSA antibodies has been detected in a group of patients affected by CHLE, including our patient, but there is a lack of evidence about the pathogenetic role [7]. It has been supposed that the Ro antigen may migrate to the keratinocyte membrane from the nucleus due to a physical trigger.

Familial CHLE is related to a mutation in the TREX1 gene, encoding for 3 '-5' repair exonuclease 1 , presenting an autosomal dominant inheritance [8]. The mutation of the TREX1 gene is responsible for CHLE, Aicardi-Goutières syndrome, and retinal vasculopathy with cerebral leukodystrophy. Interestingly, up to $40 \%$ of patients affected by Aicardi-Goutières syndrome experience CHLE-like lesions. In CHLE, a missense mutation (D18N) may be detected, but also a heterozygous p.F17S mutation. TREX1 is a DNase specific for ss-DNA (single stranded), damaged by the granzyme A protease [9]. Ravenscroft et al. [10] reported a familial case (mother and son) of CHLE presenting a heterozygous mutation in the SAMHD1 gene. Unlike sporadic CHLE, which is usually observed in middle-aged women, the familial form has an early onset in childhood and may improve with age [2].

CHLE diagnosis follows the Mayo Clinic diagnostic criteria suggested by Su et al. [1] in 1994. These contain two major criteria: (1) cutaneous acral lesions triggered by cold or damp conditions and (2) histological or indirect immunofluorescence evidence of LE, and three mi- nor criteria: (1) coexistence of other forms of SLE or CLE, (2) response to specific treatment, and (3) negative results of cryoglobulin or cold agglutinin studies.

Both major criteria and at least one minor criterion must be fulfilled. Our patient satisfied all of these criteria. In addition, we performed nail fold capillaroscopy to determine the presence of microvascular abnormalities. In fact, considering the inflammatory role in LE of new microvessel formation and endothelial cell damage, namely angiogenesis, nail fold capillaroscopy is the best method for analyzing these changes. We believe that this noninvasive technique may be very helpful in diagnosis orientation and, correspondingly, in follow-up during the treatment [11].

The antibody profile in CHLE is unspecific and does not correlate with the prognosis or development of SLE. The antibodies anti-Ro/SSA, antinuclear antibodies, and anti-phospholipid are detectable together with a rheumatic factor (half of the cases) or hypergammaglobulinemia. In our case, the patient had increased levels of $\alpha_{1^{-}}, \alpha_{2^{-}}$, and $\gamma$-globulins, together with antinuclear antibodies and Ro/SSA.

CHLE treatment is not fully standardized and the effects of conventional agents used for CLE, including antimalarial agents, have been demonstrated to be inconsistent [2]. In particular, the effect of hydroxychloroquine on CHLE is questionable, although Chasset et al. [12] recently reported a good response rate in $31 \%$ of individuals.

The first-line measure consists in the protection of acral sites from low temperatures and cold weather to prevent recurrences. The prompt treatment of necrotic lesions with topical or systemic antimicrobial drugs is crucial to prevent superimposed infections. Topical steroids have been shown to be beneficial in up to half of patients, particularly if associated with a short course of systemic steroids. Topical calcineurin inhibitors (tacrolimus ointment and pimecrolimus cream) may also be effective in preventing local side effects of prolonged topical steroids, including skin atrophy. Calcium channel blockers (nifedipine) reduce pain and erythema, due to their effect against vasoconstriction [2]. Smoking cessation may be considered an option to increase the benefits of the therapy. Several authors described successful treatment with mycophenolate mofetil, which can be considered an addon option in cases of failure of first-line therapies [13-15]. Recently, the use of fumaric acid esters (dimethylfumarate and monoethylhydrogen fumarate salts) in 2 patients, where other treatment had failed, resulted in improvement of cutaneous symptoms [16]. 
In conclusion, we reported a case of CHLE with explicit nail involvement. Vascular impairment was the direct cause of nail abnormalities, with the consequence of complete nail plate destruction. We confirm that CHLE patients respond better to symptomatic treatments, while conventional drugs are helpful in treating SLE patients, but not patients with concomitant CHLE.

\section{Statement of Ethics}

Verbal and informed consent was obtained from the patient.

\section{Disclosure Statement}

The authors have no conflict of interest to disclose.

\section{References}

$\checkmark 1$ Su WP, Perniciaro C, Rogers RS 3rd, White JW Jr: Chilblain lupus erythematosus (lupus pernio): clinical review of the Mayo Clinic experience and proposal of diagnostic criteria Cutis 1994;54:395-399.

2 Hedrich CM, Fiebig B, Hauck FH, Sallmann S, Hahn G, Pfeiffer C, Heubner G, Lee-Kirsch MA, Gahr M: Chilblain lupus erythematosus - a review of literature. Clin Rheumatol 2008; 27:949-954.

-3 Millard LG, Rowell RN: Chilblain lupus erythematosus (Hutchinson). A clinical and laboratory study of 17 patients. Br J Dermatol 1978;98:497-506.

4 Yell JA, Mbuagbaw J, Burge SM: Cutaneous manifestations of systemic lupus erythematosus. Br J Dermatol 1996;135:355-362.

5 Komori T, Otsuka A, Honda T, Kaku Y, Kabashima K: A case of chilblain lupus erythematosus with lupus erythematosus/lichen planus overlap syndrome. J Eur Acad Dermatol Venereol 2017;31:e424-e425.
-6 Arias-Santiago SA, Girón-Prieto MS, Callejas-Rubio JL, Fernández-Pugnaire MA, Ortego-Centeno N: Lupus pernio or chilblain lupus?: two different entities. Chest 2009;136 946-947.

7 Franceschini F, Calzavara-Pinton P, Quinzanini M, Cavazzana I, Bettoni L, Zane C, Facchetti F, Airò P, McCauliffe DP, Cattaneo R: Chilblain lupus erythematosus is associated with antibodies to SSA/Ro. Lupus 1999;8: 215-219.

8 Günther C, Hillebrand M, Brunk J, LeeKirsch MA: Systemic involvement in TREX1associated familial chilblain lupus. J Am Acad Dermatol 2013;69:e179-e181.

9 Prendiville JS, Crow YJ: Blue (or purple) toes: chilblains or chilblain lupus-like lesions are a manifestation of Aicardi-Goutières syndrome and familial chilblain lupus. J Am Acad Dermatol 2009;61:727-728.

10 Ravenscroft JC, Suri M, Rice GI, Szynkiewicz M, Crow YJ: Autosomal dominant inheritance of a heterozygous mutation in SAMHD1 causing familial chilblain lupus. Am J Med Genet A 2011;155A:235-237.
1 Alessandrini A, Starace M, Piraccini BM: Dermoscopy in the evaluation of nail disorders. Skin Appendage Disord 2017;3:70-82.

12 Chasset F, Bouaziz JD, Costedoat-Chalumeau N, Francès C, Arnaud L: Efficacy and comparison of antimalarials in cutaneous lupus erythematosus subtypes: a systematic review and meta-analysis. Br J Dermatol 2017;177:188-196.

13 Boehm I, Bieber T: Chilblain lupus erythematosus Hutchinson: successful treatment with mycophenolate mofetil. Arch Dermatol 2001; 137:235-236

14 Gouillon L, Debarbieux S, Berruyer M, Fabien N, Lega JC, Thomas L: Chilblain lupus erythematosus treated successfully with mycophenolate mofetil. Int J Dermatol 2017;56:e158-e159.

15 Patel S, Hardo F: Chilblain lupus erythematosus. BMJ Case Rep 2013 Nov 27; 2013.

16 Saracino AM, Orteu CH: Severe recalcitrant cutaneous manifestations in systemic lupus erythematosus successfully treated with fumaric acid esters. Br J Dermatol 2017;176: $472-480$ 